\title{
TITLE: Species' traits influenced their response to recent climate change
}

Michela Pacifici ${ }^{1}$, Piero Visconti ${ }^{2}$, Stuart H.M. Butchart ${ }^{3,4}$, James, E.M. Watson $^{5,6}$, Francesca M. Cassola ${ }^{1}$, and Carlo Rondinini ${ }^{1}$

${ }^{1}$ Global Mammal Assessment program, Department of Biology and Biotechnologies, Sapienza Università di Roma, Viale dell'Università 32, I-00185 Rome, Italy

${ }^{2}$ United Nations Environment Programme-World Conservation Monitoring Centre (UNEPWCMC), 219 Huntingdon Road, CB3 0DL, Cambridge, UK.

${ }^{3}$ BirdLife International, David Attenborough Building, Pembroke Street, Cambridge CB23QZ, UK.

${ }^{4}$ Department of Zoology, University of Cambridge, Downing Street, Cambridge CB23EJ, UK.

${ }^{5}$ School of Geography, Planning and Environmental Management, University of Queensland, Brisbane, Queensland 4072, Australia.

${ }^{6}$ Global Conservation Program, Wildlife Conservation Society, 2300 Southern Boulevard, Bronx, NY 10460, USA.

Summary: 168 words

Main text: 2054 words

Methods: 1241 words

Figure legends: 146 words

Display items: 1 table, 2 figures

References: 35 
1 TITLE: Species' traits influenced their response to recent climate change

2

While it is widely accepted that future climatic change - if unabated - is likely to have major impacts on biodiversity ${ }^{1,2}$, few studies have attempted to quantify the number of species whose populations have already been impacted by climate change ${ }^{3,4}$. Using a systematic review of published literature, we identified mammals and birds for which there is evidence that they have already been impacted by climate change. We modelled the relationships between observed responses and intrinsic (e.g., body mass) and spatial traits (e.g., temperature seasonality within the geographic range). Using this model, we estimated that $47 \%$ of terrestrial non-volant threatened mammals (out of 873 species) and $23.4 \%$ of threatened birds (out of 1272 species) may have already been negatively impacted by climate change in at least part of their distribution. Our results suggest that populations of large numbers of threatened species are likely to be already affected by climate change, and that conservation managers, planners and policy makers must take this into account in efforts to safeguard the future of biodiversity.

The rate of warming over the last 50 years $\left(0.13^{\circ} \mathrm{C} \pm 0.03^{\circ} \mathrm{C}\right.$ per decade) is nearly twice that for the previous 50 years $^{5}$, and the global temperature by 2100 is likely to be $5-12$ standard deviations above the Holocene mean ${ }^{6}$. The effects of climate change on some species are already being witnessed, with changes documented in spatial distribution, abundance, demography, phenology and morphology ${ }^{7,8}$. However, to date, no quantification of the number of species for which at least one population has been currently impacted by climate change, and the extent of these impacts, has been conducted, even for the better-studied taxa such as birds and mammals. The predominant focus of climate change assessments for species has been that of bioclimatic niche modelling, which focuses on correlative analyses between species' geographic ranges and bioclimatic variables ${ }^{9,10}$, but these studies ignore observed changes in distribution, phenology and 
abundance of species in response to contemporary climate change ${ }^{10}$. Species' life-history traits, such as dispersal and generation length, have been hypothesised to be important in determining species' sensitivity to climate change and their capacity to adapt to $\mathrm{it}^{11}$, but only a limited number of studies have so far provided evidence that animal species with certain traits are more likely than others to be adversely affected by changes in climate ${ }^{12-15}$.

In this study we first aimed at performing a meta-analysis to identify the life-history traits that confer vulnerability to climate change in birds and mammals (Supplementary Table 1). From a literature search, we identified 70 studies covering 120 mammal species and 66 studies relating to 569 bird species whose populations had (or sought evidence for) a response to climate change in recent decades. We divided this response into four categories: i) negative, if $>50 \%$ of the populations experienced reductions in one or more of the following parameters: population size, geographic range size, reproductive rate, survival rate, body mass ii) positive, if the species experienced increases in one or more of the parameters and/or adaptability to new climatic conditions, iii) unchanged, if no response was observed despite the recorded change in climate, and iv) mixed, if the species showed opposite responses of one or more of the parameters across its geographic range (Supplementary Table 2; see Methods). For all mammals and birds covered by the studies, we compiled data on selected intrinsic traits and spatial traits in order to assess quantitatively which of these are associated with negative responses to climate change. To control for the magnitude of climate change experienced, we also computed the mean difference in temperature between the present and the recent past within the geographic range of each species, treating breeding and non-breeding ranges separately for migratory birds.

By using information on the impacts of climate change in the study areas and life-history traits, we were able to identify the species whose populations are more likely to have experienced negative impacts in the regions affected by climatic changes as those described in the analysed papers. We estimated the likelihood of a species' population to have exhibited any of the four 
categories of responses to climate change with a multinomial regression model. This allowed us to test our hypotheses about the relationship between intrinsic and spatial traits and the responses of mammals and birds to climate change. Since we believe that these factors mediate the response to climate change similarly worldwide, although future studies will be crucial to test this assumption, we then predicted the likely past responses of all birds and terrestrial non-volant mammals listed as threatened in the IUCN Red List of Threatened species ${ }^{16}$. By making predictions on the species for which the levels of climatic hazard experienced are known, we provide the first quantification of the number of taxa that may have already been impacted, although further data need to be collected to say with certainty that there has been an effect on the whole species' persistence. We focused on threatened species because the vast majority are known or inferred to have declined, therefore if they are at risk from climate change there is a real chance that it has played a role in these declines, even if it was not recorded in the assessments.

For the first time we identified a relationship between a set of several variables, both intrinsic and spatial, and the response of mammals and birds to climate change, while previous studies mostly focused on a few biological traits and their relation with the type of impact ${ }^{3,4,17,18}$. In addition, we were able to provide insights into the estimation of climate change threat for poorlystudied species.

\section{Characteristics of observed and potentially impacted species}

The observed response to recent climate change was negative for $38.3 \%$ of mammals and 20.9\% of birds in our dataset (Fig. 1a). Birds and mammals in Europe and North America were the subjects of considerably more studies (54\% and 38\%, respectively) than were taxa in South America (4\%) and Oceania (2\%), and less than $1 \%$ of species in our dataset were in Africa, Asia 
and Antarctica (Fig. 2). This spatial bias implies that, for species with particular traits living in less studied continents, our findings might be less generalizable.

Mammals most at risk from climate change are those not fossorial, that experienced large changes in temperature in the last 60 years and have low precipitation seasonality within their distributions (Supplementary Table 3). In areas with reduced precipitation and/or temperature seasonality, it is likely that plant species may have narrower climatic tolerances, and therefore that these areas may have already experienced vegetation changes with consequential loss of habitat for animals living there ${ }^{19}$. A more specialized diet was also associated with greater probability of negative responses in mammals. Our findings are in agreement with previous studies on the predictors of general extinction risk ${ }^{20}$, in which species with narrower diet breadths were associated with lower ability to exploit resources and adapt to new environmental conditions and selective pressures.

For birds, negative responses in both breeding and non-breeding areas were generally observed in species that experienced large changes in temperatures in the last 60 years, live at high altitudes, and have low temperature seasonality within their distributions. Negative impacts were also associated with relatively high maximum temperature recorded within breeding areas, and low dispersal distances, longer generation lengths, reduced precipitation seasonality and restricted altitudinal ranges in non-breeding distributions (Supplementary Tables 4-5). Populations of species living at high altitudes and in colder places have fewer opportunities to move toward cooler areas or upslope to avoid increasing temperatures, and hence may have increased extinction risk. Modest shifts to higher or lower altitudes are associated with large changes in ambient temperature ${ }^{21}$, thus facilitating potential adaptive flexibility. In addition, temperature is an important determinant of laying dates of birds because higher temperatures may induce earlier laying ${ }^{22}$, and so for animals living in these environments the effects of temperature changes may have been exacerbated, potentially leading to disruption in synchronisation between the timing of chick-feeding and peak 
food availability ${ }^{23}$. Interestingly, we found that birds with longer generation times have responded less to warming. In long-lived species, the effects of climate change have probably been less evident because adaptation and range shifts occur over a longer time $\operatorname{span}^{24}$, therefore we would need to monitor the populations of these species for an extended period in order to observe any changes.

On average, it is likely that at least one population of 414 threatened mammals out of 873 species (47\%), and 298 threatened birds out of 1272 (23.4\%) has responded negatively to climate change (Fig. 1; Supplementary Tables 6-7), because they have the same combinations of traits as those species documented to have declined owing to climate change. This implies that, in the presence of adverse environmental conditions, populations of these species had a high probability of being negatively impacted by recent climatic changes.

Mammals had only 2 orders out of 11 (i.e. rodents and insectivores) that mostly benefited from recent climatic changes. Both of these orders are generally characterized by fast reproductive rates and low habitat specialization ${ }^{25}$. Moreover, most of the species in these orders are fossorial, and they may be less exposed to climate change owing to buffering of temperatures in burrows. Primates, Proboscidea and marsupials are the mammals with the highest percentage of threatened species predicted to have been negatively impacted by climate change (Table 1), and for which we are more confident about our predictions (Supplementary Table 10). Primates and marsupials are mostly concentrated in tropical areas ${ }^{26}$, most of which have had climatically stable environments during the Holocene. Therefore, many of these taxa have evolved to live within more restricted environmental tolerances and are likely to be most affected by rapid changes and extreme events ${ }^{27}$. In addition, primates and elephants are characterized by very slow reproductive rates that reduce their ability to adapt to rapid changes in environmental conditions ${ }^{13}$.

Birds showed the opposite trend, with only 3 orders out of 19 (i.e. Anseriformes, Charadriiformes and Cuculiformes) having more species with a predicted negative impact than not 
(Table 1). Most of the species included in the first two orders inhabit aquatic environments, which are considered among the most vulnerable to temperature increase due to habitat loss and fragmentation $^{28}$ and harmful algal bloom expansions ${ }^{29}$. In addition, changes in climate in tropical and subtropical forest areas, already exacerbated by habitat degradation ${ }^{2}$, may threaten forestdependent species (e.g., Cuculiformes).

\section{Conclusions}

The vast majority of assessments of species' risk from climate change have focused on future projections (e.g., ${ }^{30,31}$ ), while analyses of observed impacts to date have focused on detecting a signal of climate change rather than quantifying the number of species whose populations are likely to have been impacted. By undertaking a systematic review, we found evidence of observed responses to recent changes in climate for almost 700 species of mammals and birds. We note that only $7 \%$ of mammals and $4 \%$ of birds for which we found evidence of a negative response are coded on the IUCN Red List of Threatened Species as threatened by 'climate change and severe weather' under the 'threats classification scheme' (Supplementary Tables 8-9). While this can partly be explained by the fact that species classified as 'Least Concern' on the Red List generally have few or no threats coded, the figures we found were $11 \%$ and $31 \%$, respectively for threatened mammals and birds. This apparent mismatch is probably due to the severity of decline driven by climate change being uncertain for most species. Reasons for this include: (a) information from other parts of their distribution is not available; (b) other threats (e.g., habitat loss from agricultural expansion, overexploitation etc.) may have had a greater impact, thus masking the effects of climate; and/or (c) data on climatic trends at a local scale are difficult to obtain, making it difficult to make inferences about the threat severity. Furthermore, threats to several species remain poorly understood because the majority of threatened species live in tropical areas which are generally poorly studied and monitored ${ }^{32}$. 
Although our predictions for individual species may be subject to varying degrees of uncertainty, depending on the taxonomic order and the spatial or intrinsic trait considered, the confidence intervals around the number of species whose populations may have been negatively impacted suggest that our extrapolation is robust, especially for mammals. Improved monitoring of the abundance and distribution of those taxa identified as most vulnerable (Supplementary Figs. 12-3-4-5-6), and targeting such monitoring in areas where the effects of climate change are likely to occur soonest - particularly in the tropics - are crucial to increase empirical knowledge about climate change impacts on species, and to validate and improve projections of future impacts.

Despite these uncertainties, our results suggest that the impact of climate change on mammals and birds in the recent past is currently greatly underappreciated: large numbers of threatened species have already been impacted in at least part of their range. Given that scientific efforts in this field have largely focussed on predicting the impact of future climate change on species and ecosystems ${ }^{33}$, we recommend that research and conservation efforts give greater attention to the 'here and now' of climate change impacts on life on earth. This also has significant implications for intergovernmental policy fora such as the Convention on Biological Diversity and the Intergovernmental science-policy Platform on Biodiversity and Ecosystem Services, and the revision of the strategic plan of the United Nation Framework Convention on Climate Change. 


\section{Aknowledgments}

172 We thank Dr. Luca Santini for stimulating discussions on phylogenetic models.

174 Author contributions

175 M.P., P.V., C.R., J.E.M.W. designed the framework for the meta-analysis. M.P. conducted the 176 analyses and collected the data for mammals. P.V. contributed to the analyses. S.H.M.B. provided 177 data and examined the results for birds. F.C. collected data for birds. All authors contributed to the 178 writing, discussed the results and commented on the manuscript.

\section{Competing financial interests}

181 The authors declare no competing financial interests. 


\section{References}

1. Bellard, C., Bertelsmeier, C., Leadley, P., Thuiller, W. \& Courchamp, F. Impacts of climate change on the future of biodiversity. Ecol. Lett. 365-377 (2012). doi:10.1111/j.14610248.2011.01736.x

2. IPCC. Climate Change 2014: Impacts, Adaptation, and Vulnerability. Part A: Global and Sectoral Aspects. Contribution of Working Group II to the Fifth Assessment Report of the Intergovernmental Panel on Climate Change [Field, C.B., V.R. Barros, D.J. Dokken, K.J. Mach, M.D. Mastrandrea, T.E. Bilir, M. Chatterjee, K.L. Ebi, Y.O. Estrada, R.C. Genova, B. Girma, E.S. Kissel, A.N. Levy, S. MacCracken, P.R. Mastrandrea, and L.L. White (eds.)]. Cambridge University Press, Cambridge, United Kingdom and New York, NY, USA, 1132 pp. (2014).

3. Parmesan, C. Ecological and Evolutionary Responses to Recent Climate Change. Annu. Rev. Ecol. Evol. Syst. 37, 637-669 (2006).

4. Chen, I.-C., Hill, J. K., Ohlemüller, R., Roy, D. B. \& Thomas, C. D. Rapid range shifts of species associated with high levels of climate warming. Science 333, 1024-1026 (2011).

5. IPCC. Climate Change 2013: The Physical Science Basis. Contribution of Working Group I to the Fifth Assessment Report of the Intergovernmental Panel on Climate Change [Stocker, T.F., D. Qin, G.-K. Plattner, M. Tignor, S.K. Allen, J. Boschung, A. Nauels, Y. Xia, V. Bex and P.M. Midgley (eds.)]. Cambridge University Press, Cambridge, United Kingdom and New York, NY, USA, 1535 pp.(2013).

6. Marcott, S. a, Shakun, J. D., Clark, P. U. \& Mix, A. C. A reconstruction of regional and global temperature for the past 11,300 years. Science 339, 1198-201 (2013).

7. Parmesan, C. \& Yohe, G. A globally coherent fingerprint of climate change impacts across natural systems. Nature 421, 37-42 (2003).

8. Lane, J. E., Kruuk, L. E. B., Charmantier, A., Murie, J. O. \& Dobson, F. S. Delayed phenology and reduced fitness associated with climate change in a wild hibernator. Nature 489, 554-7 (2012).

9. Pearson, R. G. \& Dawson, T. P. Predicting the impacts of climate change on the distribution of species: are bioclimate envelope models useful? Glob. Ecol. Biogeogr. 12, 361-371 (2003).

10. Pacifici, M. et al. Assessing species vulnerability to climate change. Nat. Clim. Change 5, 215-225 (2015).

11. Dawson, T. P., Jackson, S. T., House, J. I., Prentice, I. C. \& Mace, G. M. Beyond predictions: biodiversity conservation in a changing climate. Science 332, 53-8 (2011).

12. Perry, A. L., Low, P. J., Ellis, J. R. \& Reynolds, J. D. Climate change and distribution shifts in marine fishes. Science 308, 1912-5 (2005).

13. Angert, A. L. et al. Do species' traits predict recent shifts at expanding range edges? Ecol. Lett. 14, 677-89 (2011). 
14. Poyry, J., Luoto, M., Heikkinen, R. K., Kuussaari, M. \& Saarinen, K. Species traits explain recent range shifts of Finnish butterflies. Glob. Change Biol. 15, 732-743 (2009).

15. Santini, L. et al. A trait-based approach for predicting species responses to environmental change from sparse data: how well might terrestrial mammals track climate change? Glob. Change Biol. 22, 2415-2424 (2016).

16. IUCN 2015. The IUCN Red List of Threatened Species. Version 2015-4. $<$ http://www.iucnredlist.org>. Downloaded on 18 March 2016.

17. McCain, C. M. \& King, S. R. B. Body size and activity times mediate mammalian responses to climate change. Glob. Change Biol. 20, 1760-1769 (2014).

18. Bradshaw, C. J. A. et al. Predictors of contraction and expansion of area of occupancy for British birds. Proc. R. Soc. B (2014).

19. Janzen, D. Why mountain passes are higher in the tropics. Am. Nat. 101, 233-249 (1967).

20. Gehring, T. M. \& Swihart, R. K. Body size, niche breadth, and ecologically scaled responses to habitat fragmentation: mammalian predators in an agricultural landscape. Biol. Conserv. 109, 283-295 (2003).

21. Loarie, S. R. et al. The velocity of climate change. Nature 462, 1052-5 (2009).

22. Visser, M. E., Holleman, L. J. M. \& Caro, S. P. Temperature has a causal effect on avian timing of reproduction. Proc. Biol. Sci. 276, 2323-31 (2009).

23. Visser, M., Both, C. \& Lambrechts, M. Global climate change leads to mistimed avian reproduction. Adv. Ecol. Res. 35, 89-110 (2004).

24. Vedder, O., Bouwhuis, S. \& Sheldon, B. C. Quantitative Assessment of the Importance of Phenotypic Plasticity in Adaptation to Climate Change in Wild Bird Populations. PLoS Biol. 11, e1001605 (2013).

25. Capizzi, D., Bertolino, S. \& Mortelliti, A. Rating the rat: Global patterns and research priorities in impacts and management of rodent pests. Mamm. Rev. 44, 148-162 (2014).

26. Brockman, D. K. \& van Schaik, C. P. Seasonality in Primates: Studies of Living and Extinct Human and Non-Human Primates. 44, (Cambridge University Press, 2005).

27. Corlett, R. Climate change in the tropics: the end of the world as we know it? Biol. Conserv. 151,22-25 (2012).

28. Isaak, D. \& Rieman, B. Stream isotherm shifts from climate change and implications for distributions of ectothermic organisms. Glob. Change Biol. 19, 742-751 (2013).

29. Glibert, P., Allen, J. I. \& Artioli, Y. Vulnerability of coastal ecosystems to changes in harmful algal bloom distribution in response to climate change: projections based on model analysis. Glob. Change Biol. 20, 3845-3858 (2014). 
30. Foden, W. B. et al. Identifying the World's Most Climate Change Vulnerable Species: A Systematic Trait-Based Assessment of all Birds, Amphibians and Corals. PLoS One 8, e65427 (2013).

31. Schloss, C. A., Nuñez, T. A. \& Lawler, J. J. Dispersal will limit ability of mammals to track climate change in the Western Hemisphere. PNAS 2012, (2012).

32. Jenkins, C., Pimm, S. \& Joppa, L. Global patterns of terrestrial vertebrate diversity and conservation. Proc. Natl. Acad. Sci. 110, doi: 10.1073/pnas.1302251110 (2013).

33. Visconti, P. et al. Projecting Global Biodiversity Indicators under Future Development Scenarios. Conserv. Lett. 00, 1-8 (2015).

34. Prum, R. O. et al. A comprehensive phylogeny of birds (Aves) using targeted nextgeneration DNA sequencing. Nature 526, 569-573 (2015).

35. Fritz, S. a, Bininda-Emonds, O. R. P. \& Purvis, A. Geographical variation in predictors of mammalian extinction risk: big is bad, but only in the tropics. Ecol. Lett. 12, 538-549 (2009). 


\begin{tabular}{|c|c|c|}
\hline Taxonomic order & Negative & Positive \\
\hline \multicolumn{3}{|l|}{ Mammals } \\
\hline CARNIVORA & $18(29.51 \%)$ & $35(57.38 \%)$ \\
\hline CETARTIODACTYLA & $56(59.57 \%)$ & $2(2.13 \%)$ \\
\hline DASYUROMORPHIA & $12(100 \%)$ & 0 \\
\hline DIDELPHIMORPHIA & 0 & $9(100 \%)$ \\
\hline DIPROTODONTIA & $44(100 \%)$ & 0 \\
\hline EULIPOTYPHLA & 0 & $4(4.82 \%)$ \\
\hline LAGOMORPHA & $10(55.56 \%)$ & 0 \\
\hline PERISSODACTYLA & $8(61.54 \%)$ & 0 \\
\hline PRIMATES & $199(100 \%)$ & 0 \\
\hline PROBOSCIDEA & $2(100 \%)$ & 0 \\
\hline RODENTIA & $65(19.23 \%)$ & $44(13.02 \%)$ \\
\hline \multicolumn{3}{|l|}{ Birds } \\
\hline ACCIPITRIFORMES & $8(16 \%)$ & $34(68 \%)$ \\
\hline ANSERIFORMES & $10(40 \%)$ & $8(32 \%)$ \\
\hline BUCEROTIFORMES & 0 & 0 \\
\hline CAPRIMULGIFORMES & $8(13.11 \%)$ & $21(34.43 \%)$ \\
\hline CHARADRIIFORMES & $26(57.78 \%)$ & $3(6.67 \%)$ \\
\hline CICONIIFORMES & $3(50 \%)$ & $3(50 \%)$ \\
\hline COLUMBIFORMES & $16(25 \%)$ & $47(73.44 \%)$ \\
\hline CORACIIFORMES & 0 & $17(89.47 \%)$ \\
\hline CUCULIFORMES & $6(66.67 \%)$ & $2(22.22 \%)$ \\
\hline FALCONIFORMES & $2(33.33 \%)$ & 0 \\
\hline GALLIFORMES & $22(29.33 \%)$ & $3(4 \%)$ \\
\hline GRUIFORMES & $13(29.55 \%)$ & $29(65.91 \%)$ \\
\hline PASSERIFORMES & $171(30 \%)$ & $112(19.65 \%)$ \\
\hline PELECANIFORMES & $6(31.58 \%)$ & $8(42.11 \%)$ \\
\hline PICIFORMES & $5(14.71 \%)$ & $17(50 \%)$ \\
\hline PODICIPEDIFORMES & $1(25 \%)$ & $1(25 \%)$ \\
\hline PROCELLARIIFORMES & 0 & $8(13.56 \%)$ \\
\hline PSITTACIFORMES & 0 & $103(99.04 \%)$ \\
\hline SPHENISCIFORMES & $1(10 \%)$ & 0 \\
\hline STRIGIFORMES & 0 & $6(13.95 \%)$ \\
\hline SULIFORMES & 0 & $10(100 \%)$ \\
\hline
\end{tabular}

Table 1: Predicted responses of threatened species in different taxonomic orders to climate change.

Positive responses were assigned to species that benefited from recent climatic changes. Percentages indicate the proportion of threatened species for each type of response. 
Figure 1 | Observed and predicted response of mammals and birds to climate change. a) Red bars show the percentage of species whose populations were documented to have had, or are predicted to have had, a negative response to climate change in the study period (studies spanned from 1858 to 2010); green bars represent the percentage of species with a positive response; blue bars indicate the percentage of species with no response; orange bars show the percentage of species with mixed responses. b) Bars with the number of species whose populations had an observed response to climate change are coloured in white, while those used for predictions are shown in black.

Figure 2 | Map of the study sites. Circle size represents the number of bird (blue circles) and country. 


\section{Methods}

Using ISI Web of Knowledge we conducted a systematic literature search of all relevant articles - published between 1990 and 2015 - that i) reported an observed change in climate in the study area, ii) indicated that birds and/or mammals have undergone a change (e.g., in distribution, population size, phenology, behaviour, genotype, phenotype) attributable to climate in the past 100 years, and/or iii) suggested that populations of a species were not affected by recent climate change. For each study and each species considered (70 studies and 120 species for mammals, 66 studies and 569 species for birds), we identified the type of impact experienced.

A negative response was assigned to a species if all (at least one) or $>50 \%$ of its populations (if the species had both negative and no responses in different portions of its range) were reported to have undergone declines in population size, geographic range size, survival or reproductive rate, and body mass, thus reducing the risk of false attributions. These responses were confidently attributable to recent climate change by the authors of the studies, for instance due to the fact that the most significant change in environmental and biotic conditions reported in the area in which the population of the species was impacted was related to climatic variables. Although we acknowledge that some of the studies may have been more rigorous than others, with such variation in the methods used and the effect size themselves it would have been difficult to adjudicate the level of confidence around the claimed relationship, although we believe that evaluating the strength of attribution is a priority for future work.

A positive response was assigned if the majority of the populations of a species experienced geographic range expansions, increase in population size, survival rate and/or reproductive rate, body mass, and/or changes in phenology. An unchanged response was attributed if no response was observed despite the recorded change in climate. Finally, species that exhibited a combination of the negative and positive (not necessarily in the same proportion) responses in different parts of their range were classified as mixed. 


\section{Statistical analysis}

To identify the relationships between the observed response of mammals and birds to climate change and a set of intrinsic and spatial variables (see Supplementary Methods for description of these predictors and a priori hypotheses), we performed a multinomial logistic regression using the 'nnet' package in R. This model uses maximum likelihood estimation to evaluate the probability of the different possible outcomes of a categorical dependent variable with more than two classes. In order to reduce the overdispersion in models and avoid collinearity, we performed Spearman's correlation tests between the predictors and removed those that were highly correlated $\left(\mathrm{R}^{2}>0.75\right)$ and led to the minimum loss in model performance.

We included taxonomic order as fixed variable of our models, for a total of 11 orders of mammals and 22 of birds. By including taxonomy as a fixed effect, we aimed to control for the nonindependence of observed responses across species, and for the latent variables that may affect the responses to climate change that are phylogenetically conserved. We did not include taxonomic family or genus because it resulted in strong underdispersion, as observed data on the response to climate change (which we used as a base for our predictions on threatened species) were often only available for the populations of one species per family/genus. Since we are not aware of frequentist methods to implement phylogenetically corrected models with a multinomial distribution, and concerned that phylogenetic non-independence in the species in our dataset could nevertheless be important, we tested for the existence of phylogenetic signal in the residuals of our models. We used phylogenetic trees for mammals and birds ${ }^{34,35}$ to estimate Pagel's lambda, assuming a starshaped phylogeny and the actual phylogeny (Brownian motion models). We tested whether the value of lambda differed significantly from 0 (no phylogenetic signal) and 1 (trait distribution matches a Brownian model of evolution), by computing the likelihood ratio, and then comparing it to a Chi-squared distribution with one degree of freedom. If the test is significant there is phylogenetic signal in the residuals. However, we found lambda values of $6.73 \mathrm{e}-05,5.56 \mathrm{e}-04$ and 
non-breeding ranges, respectively. Therefore we conclude that there is no phylogenetic signal in the residuals of the models and a phylogenetically-informed model is not justified.

We performed a model selection using the AIC to find the set of predictors to include in the final model that minimize the Kullback-Leibler distance between the model and the observed values. We applied logarithmic and quadratic transformations to the predictors and included variable interactions in the models, but most of them did not lead to a decrease in AIC or increase in model performance calculated by using the Area Under the Curve (AUC). Finally, to test our models for overdispersion, we calculated the sum of squared Pearson residuals and compared it to the residual degrees of freedom by using a Chi-squared test. P-values close to 1 indicate that the probability of the model being overdispersed approaches 0 (Supplementary Table 13).

On the basis of the relationship between the observed response of species and our independent variables found with the best multinomial models, we predicted the probabilities of the four classes of response to climate change by using the function predict in R. For predictions we considered all threatened birds (1272 species, as listed on the 2014 IUCN Red List) and terrestrial non-volant mammals (873 species) with available data. We excluded sea mammals from our analysis because the environmental variables that influence the persistence of marine and terrestrial species are different, and most of the variables important for marine species (e.g., sea temperature, salinity) were not available for the study period. Chiroptera could not be considered in this study because of the paucity of data available on their life history.

Our model is at the species level, but our data (observed responses to climate change) is at the population level. Because the spatial extent of the study area was not available for the vast majority of studies, we were forced to average the annual temperature change experienced by the species across all of its range. However, the average climatic change might not be representative of the change experienced by the populations we used to train the model, especially with species with large range size. By resampling the response category assigned to each species from the 
367 multinomial distribution 100 times and deriving coefficient intervals and mean values of the

368 richness of species with negative responses, we tried to reduce the uncertainty around our

369 predictions. In addition, to identify the taxonomic orders for which our predictions were most

370 reliable, we used a Kolmogorov-Smirnov nonparametric test which quantifies the distance between

371 the empirical continuous distribution functions of two samples, and the null hypothesis is that the

372 samples are drawn from the same distribution. By comparing the distribution of the same numeric

373 trait in both the observed and the predicted sample, if the p-value of the test is above the $\alpha$

374 threshold, i.e. 0.05 , we can assume that threatened species in the considered taxonomic order are

375 well represented in the sample of observed data. This means that, for this order, our predictions are 376 more robust.

377

378 Data availability

379

The authors declare that [the/all other] data supporting the findings of this study are available within the article and its Supplementary Information files. 
Supplementary Table 1 | Selected potential correlates of extinction risk associated with climate change.

Supplementary Table 2 | Negative observed impacts on species.

Supplementary Table 3 | Threatened mammal species identified from models as likely to have already been negatively impacted by climate change.

Supplementary Table 4 | Threatened bird species identified from models as likely to have already been negatively impacted by climate change.

Supplementary Table 5 | Coefficient estimates, standard errors and confidence intervals of the most important predictors resulting from the best multinomial model in mammals.

Supplementary Table 6 | Coefficient estimates, standard errors and confidence intervals of the most important predictors resulting from the best multinomial model in birds (breeding range).

Supplementary Table 7 | Coefficient estimates, standard errors and confidence intervals of the most important predictors resulting from the best multinomial model in birds (non-breeding range).

Supplementary Table 8 | Response of mammals to climate change by taxonomic order.

Supplementary Table 9 | Response of birds to climate change by taxonomic order.

Supplementary Table 10 | Numeric predictor variables and orders for which our predictions on threatened species are most reliable for mammals.

Supplementary Table 11 | Numeric intrinsic and climatic predictor variables and orders for which our predictions on threatened species are most reliable for birds.

Supplementary Table 12 | Numeric spatial predictor variables and orders for which our predictions on threatened species are most reliable for birds.

Supplementary Table 13 | Results of Chi-squared tests for overdispersion.

Supplementary Table 14 | Results of a binomial model having "negative" and "non-negative" classes for the response variable of mammals.

Supplementary Table 15 | Results of a binomial model having "negative" and "non-negative" classes for the response variable of birds (breeding areas).

Supplementary Table 16 | Results of a binomial model having "negative" and "non-negative" classes for the response variable of birds (non-breeding areas).

Supplementary Table 17 | Number of studies/populations per mammal species.

Supplementary Table 18 | Number of studies/populations per mammal species.

Supplementary Figure 1 | Richness map of impacted mammals. 
414 Supplementary Figure 2 | Richness map of threatened mammals.

415 Supplementary Figure 3 | Richness map in breeding ranges of impacted birds.

416 Supplementary Figure 4 | Richness map in non-breeding ranges of migratory impacted birds.

417 Supplementary Figure 5 | Richness map in the breeding ranges of threatened birds.

418 Supplementary Figure 6 | Richness map in non-breeding ranges of threatened migratory 419 birds.

420 
a.

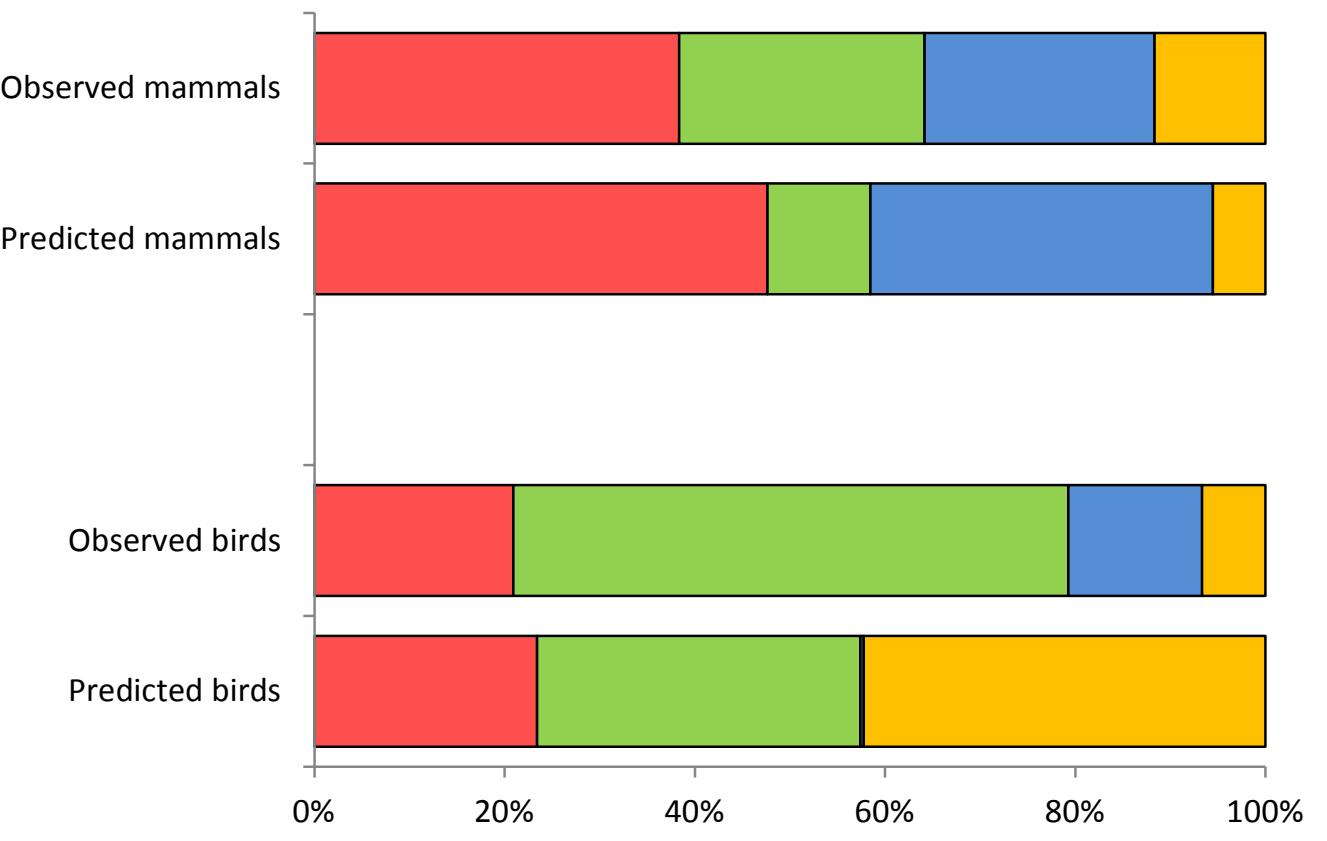

b.

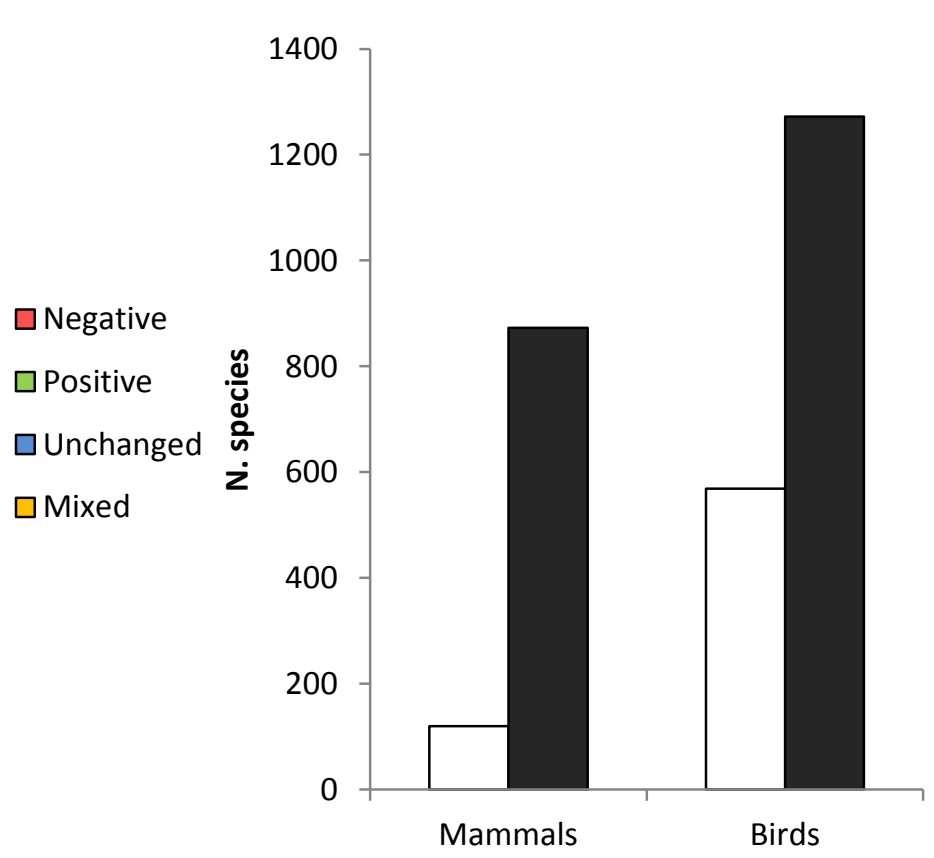

$\square$ Observed

$\square$ Predicted 


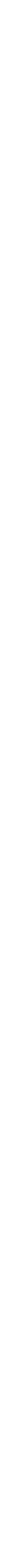

